\title{
THREE CRITICAL CHALLENGES FOR MODELING AND SIMULATION IN HEALTHCARE
}

\author{
Terry Young \\ Julie Eatock \\ School of Information Systems, \\ Computing and Mathematics \\ Brunel University \\ Uxbridge, UB8 3PH, UK
}

\author{
Mohsen Jahangirian \\ Aisha Naseer \\ School of Information Systems, \\ Computing and Mathematics \\ Brunel University \\ Uxbridge, UB8 3PH, UK
}

\author{
Richard Lilford \\ Public Health, Epidemiology \\ and Biostatistics \\ University of Birmingham \\ Birmingham B15 2TT, UK
}

\begin{abstract}
By most measures, the adoption of modeling and simulation techniques in healthcare service development falls well short of the uptake of such techniques evident in other sectors, such as business and commerce or aerospace and the military. The question is, why? To answer this, we consider three questions and then turn to the nature of answer which might lead towards greater adoption. The first is the vexed question of how good is good enough? The second concerns how best modeling should link through to decision-making; and the third concerns the culture needed to make the most of modeling and simulation (and whether it is worth the effort to make the transformation). From these, we draw an agenda for further enquiry in terms of stakeholders, their culture, data, and expectations, and the case in terms of value.
\end{abstract}

\section{INTRODUCTION}

At first sight, healthcare would appear an obvious candidate for the use of modeling and simulation . Service delivery is highly complex, often safety critical, and both the supply and demand sides involve scales of millions of people, costing trillions of dollars around the world.

Moreover, it is difficult to obtain a clinical qualification of any sort without some encounter with role-play or assessed simulations with, for instance, mannequins, which are currently the most widely used simulation-based training tool in clinical settings, costing around $\$ 40,000$. Other kinds of simulation-based training methodologies are described in Binstadt et al. (2007). Some of these can be very sophisticated. An example might be the range of training aids available for training surgeons in minimally invasive surgery. Continuing education relies upon such techniques, augmented at times, by on-line learning, and even fully integrated medical training simulation models with an "all at once" implementation (Binstadt et al. 2007). Rosen (2008) and Gaba (2004) look at the literature on simulation-based medical training, from historical and predictive perspectives, respectively.

One might have thought that, on both the demand side (having exquisitely difficult services design challenges) and on the supply side (a rich experience of acted or simulated experiences), the community would embrace the use of computerbased modeling and simulation for service design, delivery, acceptance trials, troubleshooting, and the like.

The surprise is that it does not. In a recent review (Brailsford et al. Accepted) comparing the use of modeling and simulation in healthcare with the norms in (business and manufacturing) commerce and defense (military and aerospace), only $8 \%$ of papers from healthcare reported analysis of a real problem with high levels of user engagement, compared to $36.5 \%$ in the defense literature, and $48.9 \%$ in commerce. Meanwhile the proportion of the literatures devoted to concept papers or methodological development, was only $8.1 \%$ in commerce, against $44.2 \%$ in defense and $52.3 \%$ in healthcare. By other standards, too, healthcare lags other sectors - for instance in the range of techniques or the maturity of the literature (Jahangirian et al. 2009)

To understand the situation better, a group of universities in the UK (Brunel, Cambridge, Cardiff, Southampton and Ulster) secured a grant from the Engineering and Physical Sciences. The resulting wide-ranging research study, RIGHT (Research Into Global Healthcare Tools), has been exploring the use of modelling and simulation from a number of perspectives.One of the first exercises was to try to set the adoption of these methods in healthcare against a backdrop of their usage in two other sectors - commerce and the military. This work is reported elsewhere, but as far as we are aware, this is probably the broadest survey reported, covering both the techniques adopted and the applications in which they are used. This is in 
contrast to much of the review literature which tends either to address specific modeling techniques or specific areas of application.

Table 1 lists the top five modeling and simulation techniques (Naseer, Eldabi and Jahangirian 2009; Jahangirian et al. Article in Press)

Table 1: Top 5 modeling and simulation techniques reported in the literature

\begin{tabular}{ccc} 
Commerce & Defense & Healthcare \\
\hline Discrete Event Simulation & Distributed Simulation & Discrete Event Simulation \\
System Dynamics & Discrete Event Simulation & Monte Carlo Simulation \\
Hybrid Simulation & System Dynamics & System Dynamics \\
Agent Based Simulation & Real Time Simulation & Agent Based Simulation \\
Monte Carlo Simulation & Monte Carlo Simulation & Distributed Simulation
\end{tabular}

Modeling and simulation are familiar at the policy level, although the need to publish or even the desirability of doing so may have skewed the literature. Royston (2005), for example, reports on many uses of healthcare modeling and simulation, including in governmental decision-making and the roll-out of policy. More critically, in the UK, the National Institute for Health and Clinical Excellence (NICE) relies heavily on models in issuing its guidance for NHS adoption of pharmaceuticals and medical devices - often Markov models and decision trees - to support its economic evaluation.

In terms of the main techniques and applications, the literature is huge and growing ( $>30$ papers/day in the Ovid database). The scene has been set by three reviews (Tunnicliffe Wilson 1981; Jun, Jacobson and Swisher 1999; Fone et al. 2003) Perhaps unsurprisingly, most of this process modeling has taken place in those parts of the system that are most accessible Accident \& Emergency (Emergency Room), clinics and bed management. Table 2 shows the top applications of modeling and simulation in healthcare, commerce and the military (Naseer, Eldabi and Jahangirian 2009; Jahangirian et al. Article in Press)

Table 2: Top 5 modeling and simulation applications reported in the literature

\begin{tabular}{ccc} 
Commerce & Defense & Healthcare \\
\hline Operational Management & Operational Management & Operational Management \\
Policy & Planning & Research and Development \\
Planning & Workforce/Training & Planning \\
Workforce/Training & Behavior & Policy \\
Research and Development & Risk & Quality \& Evaluation
\end{tabular}

Given that the healthcare community has some experience of artificial environment in training, that elements of the system - such as NICE - make intensive and extensive use of modeling, the question of why healthcare has not adopted what are the standard tools of the trade in other sectors is an interesting question. Harper and Pitt (2004), for instance, list some critical factors (relating their findings principally to the UK's National Health Service): the scale, complexity and change characterizing the service; diversity within its management; the lack of buy-in from key communities together with a lack of evidence to generate credibility in their thinking; the conflicting objects of the public health service; and finally a host of issues around the availability and relevance of high quality data.

In the UK, these questions have reached a certain criticality because of the new innovation focus of the Darzi review (Department of Health 2008), with its emphasis on eight 'models of care' from birth to end of life. The accent is clearly on finding better ways to deliver higher quality more consistently. A critical - and from this perspective, highly encouraging side-effect has been the intense search for system level tools throughout the delivery communities.

This paper takes a reflective approach, examining three key questions that have resisted definitive solutions for some time - and remain perplexing still. The purpose of this paper is to re-focus and shed what new light has emerged from the findings of RIGHT, most of which are being published separately. The RIGHT research has involved: 
- A series of wide-ranging literature studies into modeling and simulation in three fields (healthcare, commerce, the military), management techniques in healthcare and commerce, frameworks and stakeholders.

- A set of exemplars - engagement with real service providers that might require modeling and simulation. The sample was purposive and included both planned collaboration and opportunistic work. Only a minority of these exemplars were "successful" in the traditional sense that the modeling was undertaken to fully mutual benefit and the findings adopted. However, by bearing all the experiences in mind we hope to build up a more realistic view of what really happens in the interaction between a user community and modelers or a model.

- Built a selection tool (Naseer, Eldabi and Young Accepted) for members of the care community to use in choosing an appropriate method when making decisions.

This paper attempts to take a high level view of adoption issues around modeling and simulation, using the RIGHT findings as background, and by appealing to three case studies, provided in the next section. The questions we address in later sections are:

- How good is good enough?

- How is modeling linked to the decision-making?

- What are the other cultural barriers?

\section{THREE ILLUSTRATIVE EXAMPLES}

\subsection{Case Study 1: Bed Management In A Hypothetical Hospital}

In the mid ' 90 s, a piece of work on bed management in the UK was commissioned. From the memory of the commissioner and one of the participants, the cost was less than $£ 100 \mathrm{k}$ and the study took less than a year - perhaps 8 months - to complete. The study involved an hypothecated hospital and was conducted using DES and part of the work led to a paper in the BMJ (Bagust, Place and Posnett 1999), which established a rule of thumb - namely, that bed occupancy above $85 \%$ carried risk and that when it exceeded $90 \%$, a hospital could expect 'regular' shortages and 'periodic bed crises.' This highly cited paper (121 citations on Google Scholar, analyzed and corrected for duplications, June 11 2009) resulted from a national commission, not a specifically operational need from one hospital.

The impact has been to give bed managers across the country, and over many years, a simple benchmark by which to assess their risks and manage throughput. Both the way in which this result has been generalized and reduced to a simple rule of thumb, have enabled the findings to make an impact on the real world.

\subsection{Case Study 2: Business Planning Of Healthcare Service Offerings}

In 2005, BT set out to evaluate a set of IT-based services that they might offer into healthcare. To support their business planning, they built an SD model of a population of 100 patients. The study took around 5 months and involved a team of 56 people (Green and Young 2008). The goals of the study were to quantify the benefits of the ICT products and services, to explore the incentives in the system that might lead NHS clients to take up the putative products and services, and then to explore the budgets that those customers had.

The main result of this study was to identify features of the commercial landscape that had not been visible before namely that those with the greatest need of IS products and services had no budget, while those with budgets were not incentivised to adopt such solutions. This timely finding saved the company from losing a great deal of money on a hopeless launch campaign, but although the author knows of efforts to promulgate the news of this disconnect, it is not clear that how or ever whether the message has become embedded in the system.

\subsection{Case Study 3: An Accident And Emergency Department Model In A Real Hospital}

From September 2008 to May 2009, the RIGHT Team built a DES model of the A\&E department of a local District General Hospital. Although the engagement started with an enquiry about the sort of technology that might help all staff to share a common operational awareness about patients passing through the department, the target became to provide a basis for decision-making in streaming patients.

There were several unexpected challenges, including communications between the clinicians and the hospital teams, but especially in trying accurately to model the special measures taken by staff to meet a 4 hour throughput target. While the hospital's patient administration was able to provide lots of information, the incompleteness of some of this also took time to unravel. We expect to have published this work shortly in time for the conference, but the key element here is that obtaining 


\section{Young, Eatock, Jahangirian, Naseer and Lilford}

a baseline study that took in real admissions and provided a pattern of discharges and hospital admissions that conformed to the records took much longer than initially anticipated.

For the stakeholders, there was little to show for this delay, apart from building some confidence in the system (it was the academic side who were keen on this rigor). During this time, two experiments in reflowing patients had been completed by the hospital by the time the validation had been completed. One of these was readily replicated with the validated model and as of writing, the clinicians are keen to explore other measures.

The interesting challenge thrown up by this study was the conflict of timescales (weeks as opposed to months) between those seeking operational results to inform their decision-making and those undertaking the research. A shorter study might well have failed to replicate the basic throughput metrics, so that the clinicians would have had little cause to believe the results. On the other hand, the detailed work of building a complex model - the most complex DES model we have found to date in the literature for an A\&E department, meant that demand ran out of synch with supply.

Discussions around the modeling indicated:

- That some rules of thumb may be possible. For instance, the model was able to predict at the time of admission, what the probability of a breach of the 4-hour limit would be. Clearly, this prospective view of breach would be extremely useful and, with further analysis might help departments to monitor crowding against some simple thresholds. From an OR perspective, it would appear that clinical interventions tend to come quite late in the day (i.e. as patients approach their fourth hour) whereas general management theory and queuing theory would tend to suggest intervention should occur as soon as a breach was likely.

- That the messages from the study needed to be passed on up through the hospital management, and steps are in train to make the study and its findings more widely available. Crucially, there is significant evidence that the bed management of the hospital as a whole has a profound effect upon the responsiveness of A\&E (Cooke 2003) and that A\&E management is about the entire system and not just one or two elements of it.

- Developing this theme, discussions with the clinicians also indicated that A\&E admissions were highly sensitive to changes in health and social provision well away from the hospital. The clinicians noted, for instance, that admissions tended to rise in April (the start of the new financial year) as service cuts or changes were implemented elsewhere. The pressure on A\&E departments in the UK means that even small increases in demand rapidly convert into throughput challenges and directly on into the performance measures of the department, affecting patient care, often adversely.

\section{HOW GOOD IS GOOD ENOUGH?}

Modeling and simulation are often sold as useful for 'operational' improvement and redesigning processes. The challenge here is to perform enough modeling to yield helpful findings within the decision-making cycle of the critical stakeholders. This raises the question of what that community needs in terms of sufficient detail to find the analysis compelling, but also enough new knowledge to inform the next stage of decisions. Alternatively, models that take a strategic stance do not suffer from this restriction. The results can be published and adopted whenever needed.

Under the RIGHT program, we have followed 10 exemplars through to better understand the interaction between modeling and simulation and the business of delivering care. The three cases listed above are exceptional in that many - perhaps the vast majority of - attempts to engage end in failure to make a difference to the care delivery system. The question of the time needed by the modeling as opposed to the time available on the part of the decision-maker appears to be a common theme.

Pidd and Robinson (2007), independently, and together, as the citations contained within this reference show, have given thought to this problem. They conclude by making the 'fit' in terms of various spectra - linear representations in which the complexity generally develops from left to right. However, they also put together a two dimensional tool which links the mode of use (formal experimentation, exploratory experimentation, and facilitative use) against applications (software engineering, visual interactive modeling and participative modeling).

Taking this approach, the challenge is to match the complexity of the model to problem in hand. Even given this, there is still a question as to whether the model can deliver what the customer needs. The issue is the extent to which 'operational' modeling can overcome the challenge of delivering sufficient detail within a sufficiently short window to provide confidence that the complexity of the system in view has been modeled at an appropriate level, and also to provide meaningful inputs to the decisions being considered. The authors are aware of few, if any, hard guidelines or rules of thumb by which the modeling itself might be made operational. Given the scale and complexity of most care delivery systems, not to mention the difficulty in drawing an effective boundary around the subsystem to be modeled, it would be helpful to find some measure or 'traffic light' system that might guide the adoption of the modeling. The conventional approach, which is to rely upon the judgment of the modeler suffers from the variation from modeler to modeler and the fact that on the whole, the person under- 


\section{Young, Eatock, Jahangirian, Naseer and Lilford}

taking the modeling is likely to be more aware of the need to finesse the model towards perfection, and so the client, usually more fixed on the results, is dependent upon the view of someone whose judgment is likely to be biased in the opposite direction.

The possibility of simulation models that were in some way generic, but could then be tailored on short timescales to represent the local system, is one way of overcoming this impasse. Using such an approach, a set of four or five generic A\&E layouts might be made accessible to the community, each of which could then be populated with admission figures, resources, rotas and other elements specific to the locale.

Another approach would be to resort to a simpler model. The difficulty of doing so in the third case study, for instance, is that one critical problem lay in understanding how patients were managed just prior to a breach. There is a great deal in the literature about A\&E throughput (e.g. Cooke, Wilson and Pearson 2002; Asplin, Flottemesch and Gordon 2006; Cronin and Wright 2006; Kumar and Shim 2007; Hoot et al. 2008) but relatively few address the impact of special measures to meet a deadline or the way in which it distorts the discharge profile (Locker and Mason 2005; Mayhew and Smith 2008) and we are unaware of any attempts to model this profile.

The most obvious means of ensuring that the findings are adopted is to retrieve some general or strategic finding that can be embedded as a rule of thumb. In busy environments, rules of thumb work well with the intuition of practitioners and provide a ready guide to practice. This in turn raises interesting questions about how well the modelers can know that elements of a given study may be more widely applicable. However, to the extent that 'strategic' findings can be converted into rules of thumb and so embed themselves in practice, they represent perhaps the most obvious means of exploiting modeling and simulation in healthcare.

Thus while there is a rich literature, the question of fitting the model to the needs of the community remains unresolved and addressed generally in an ad hoc manner. It would be interesting to see whether the healthcare OR community might rise to this as a challenge and produce some helpful, viable guidelines that worked for the clinical and managerial communities.

\section{HOW IS MODELING REALLY LINKED TO THE DECISION-MAKING?}

Brailsford et al. (Accepted) describe in some detail the lessons learned through the RIGHT program around stakeholder engagement. As she notes, there are significant difficulties in identifying the relevant stakeholders quite apart from the challenges already alluded to of keeping them on board. As noted, there are no obvious, accepted taxonomies of stakeholders in modeling and simulation nor is their relationship to the decisions particularly clear. Moreover, a wider framework that might connect a series of perspectives together and provide guidance on whom to engagement with and how, has generally proved elusive (Bolt, Connell and Klein 2009; Morris and Clarkson 2009). RIGHT has explored several ways of classifying stakeholders, including the scheme presented by Brailsford et al. (Accepted): Ownership, legitimacy, power, urgency, centrality, time, money, and data. Thus far, it appears that the literature is unhelpful in providing a consensual view of who the critical stakeholders might be nor does it satisfactorily explain how their relationship to the simulation connects to their responsibility for the decision. However, there are further nuances to the problem.

Appeal to the examples above provides some insight into this problem. In the first case, the modeling was done within a very simple stakeholder relationship - a commissioner and a team of modelers. The decision-makers were not directly involved in the traditional sense. However, by producing a rule of thumb, the study enabled many decision-makers - typically operational bed managers - to apply the results. This introduced the concept of the 'absent' stakeholder, which we believe to be a poorly explored concept in modeling. Moreover, far from being a 'bad thing' (as might be expected), the decisionmakers have direct access to an output. Moreover, had a traditional approach of assembling a representative body of bed managers been followed, it is unlikely that such an elegant study could have been conducted so quickly or economically.

The 'absent' stakeholder is a recurring theme. In the case of the business development team, the decision-makers (i.e. the company or business unit board) would not usually engage closely with the modeling. However, it is clear that the findings were interpreted to that higher level and used in two ways: firstly, in a business decision not to pursue that product and service strategy; and secondly, to take out a message to policy-makers in healthcare systems that there was a strategic problem if they wished to see any uptake of such products and services. We do not have the details of the relationships between all of the players in Case Study 2, but it is not hard to see how relatively straightforward lines of command, allied to a common view of the criticality of profit-making, led to a ready connection between the model and the decision to back away from the market.

A critical element in the A\&E case study, once the model could replicate the patient administration systems discharge patterns, was to take the message on up through the system. This is one example of how the high levels of connectedness and complexity of care delivery systems make a stakeholder analysis very difficult. Since so many systems converge around $\mathrm{A} \& \mathrm{E}$, and specifically, since many of the hospital systems either impact on or are impacted by, A\&E throughput perfor- 


\section{Young, Eatock, Jahangirian, Naseer and Lilford}

mance, it would be almost impossible to gather all the stakeholders together before initiating a study. On the other hand, as findings become clearer, one of the first instincts of the local stakeholders is to elevate the findings up through the system.

Thus, as well a needing a better taxonomy of stakeholders, the simulation community needs a clearer view of which are necessary and which are not at the various stages of modeling. The general encouragement to the modeling and simulation community to engage with stakeholders, misses the reality that in many cases the decision-makers will not be accessible until or unless there is an element of success to take forward into a decision.

\section{WHAT ARE THE OTHER CULTURAL BARRIERS?}

In his book, Winning the next war, Rosen (1991) explores how the US navy started to war game with fleets of five carriers against fleets of four carriers in the early '20s. An aircraft carrier was an innovation that could not be achieved through incremental improvement or by combining existing resources. It was a totally new way of warfare and, indeed, in World War II, fleets fought complete battles without either admiral catching sight on the other, using hulls that were laid down as a result of that war-gaming and the experiments that followed. The military has both the time and indeed the need to game out new technologies and doctrines because of the high cost of both failure and, to a lesser extent, the equipment itself. Moreover, the culture supports lots of training, practice and simulation outside of the theatre of war. (In fact, an interesting theme of Rosen's monograph is whether innovation takes place better under the pressure of war - where demand is high but resources are stressed - or in peacetime, when the situation is generally reversed.). Similarly, in commerce, there is a culture of simulation for key decisions and operational improvement, outside of or even alongside business as usual.

The same does not seem to be the case for healthcare. Whether it is the sheer pressure of trying to keep the system running or the need to migrate slowly away from known, and safe, procedures when developing new pathways or processes, is not clear. However, the same cultural 'hooks' that make modeling and simulation attractive to modelers in the military and in commerce, appear to be missing or at least less well developed in healthcare. And this represents the third major challenge, namely understanding the cultural context. This challenge is critical not because it may preclude the use of such tools, but because addressing it is likely to place constraints around the type of tolls, interfaces, standards and data that are acceptable to the community. Under the next phases of RIGHT, we hope to undertake extensive fieldwork around the expectations of healthcare communities and their use of such tools. This attempt to support the process-oriented approach is supported by Graban (2008), who notes that "Toyota gets outstanding results with average people working in brilliant systems, while most other companies get mediocre results by hiring brilliant people to fight their way through broken systems."

\section{CONCLUSIONS}

If modeling and simulation are to make a greater impact on healthcare, it appears that strenuous efforts will be needed in terms of 'reducing to practice.' This will require guidelines or rules of thumb that are generally more prescriptive than exist in the literature today. In this paper we have synthesized the recent experiences of a project to explore the use of such techniques in healthcare and explored some of the findings with reference to three examples, or (brief!) case studies. Our findings are that clearer and more general guidelines are needed in three areas.

The first of these concerns the question of how good is good enough? There are some intriguing and insightful leads in the literature and the challenge now is to classify in terms of operational and strategic decisions and to provide accessible and viable guidance about how best to match the need for input to the provision of findings. In particular, new strategies may be needed to synchronize the delivery of results to the pace of decision-making in real hospitals. One option, for instance, might be to create meta-models that cover a range of designs (for instance, of A\&E departments), enabling managers to tailor the model rapidly to their specific needs quickly, and model appropriately on short timescales. The second issue concerns the classification of stakeholders so that modelers and their client communities (assuming such a modus operandi emerges) are able to assemble appropriate teams that can carry the findings forward into the decision-making. One issue raised in this paper is the extent to which 'absent' stakeholders may be an essential part of the calculation and strategies to manage both their absence and the interpretation of relevant findings to them are still needed. However, without a clearer idea of who the stakeholders are and when they need to be drawn in or to drive the decisions, it is hard to see how modeling and simulation will ever become embedded in the community. Finally, there are questions of culture and the differences between healthcare and commerce or the military, which need clarification if appropriate interfaces, standards, iconography, and so forth, are to meet the needs of clinicians and healthcare managers. In principle, these needs could be met through three taxonomies - one linking models to problems, one linking stakeholders in the modeling to stakeholders in the decisions, and one addressed the context in which modeling and decisions are mode. However the problems are addressed, we find this an exciting research agenda and plan to pursue it, along with other critical issues in the next phases of RIGHT. 
Young, Eatock, Jahangirian, Naseer and Lilford

\section{ACKNOWLEDGEMENTS}

We thank Drs Sivakumar and Sweatman, and also Claire Picton, all of The Hillingdon Hospital NHS Trust, for discussions around the material in this paper and (CP) for comments upon it. We also thank Professors Sally Brailsford and Con Connell, as well as Dr Tillal Eldabi for helpful comments. We are grateful for the support of the Engineering and Physical Sciences Research Council (EPSRC) under the MATCH (GR/S29874/0, EP/F063822/1and EP/GO12393/1 RIGHT (EP/E019900/1) grants, and for the support and input from the other members of the RIGHT team.

\section{REFERENCES}

Asplin, B. R., T. J. Flottemesch and B. D. Gordon. 2006. Developing models for patient flow and daily surge capacity research. Academic Emergency Medicine 13:1109-1113.

Bagust, A., M. Place and J. W. Posnett. 1999. Dynamics of bed use in accommodating emergency admissions: stochastic simulation model. British Medical Journal 319:155-158.

Binstadt, E. S., R. M. Walls, B. A. White, E. S. Nadel, J. K. Takayesu, T. D. Barker, S. J. Nelson and C. N. Pozner. 2007. A comprehensive medical simulation education curriculum for emergency medicine residents. Annals of Emergency Medicine 49:495-504.

Bolt, T., N. A. D. Connell and J. H. Klein. 2009. Examining the stakeholder landscape to support health policy OR projects. RIGHT Working Paper (www.right.org.uk).

Brailsford, S. C., C. Connell, J. H. Klein and B. Patel. Accepted. Stakeholder engagement in health care simulation. In Proceedings of the 2009 Winter Simulation Conference, M. D. Rossetti, R. R. Hill, B. Johansson, D. A. and I. G. Eds, Austin, Texas, Institute of Electrical and Electronics Engineers, Inc.

Cooke, M. W. 2003. Reforming the UK emergency care system. Emergency Medicine Journal 20:113-114.

Cooke, M. W., S. Wilson and S. Pearson. 2002. The effect of a separate stream for minor injuries on accident and emergency department waiting times. Emergency Medicine Journal 19:28-30.

Cronin, J. G. and J. Wright. 2006. Breach avoidance facilitator - managing the A\&E 4-hour target. Accident and Emergency Nursing 14:43-48.

Department of Health. 2008. High Quality Care For All Available at http://www.dh.gov.uk/prod_consum_dh/groups/dh_digitalassets/@dh/@en/documents/digitalasset/dh_085828.pdf

Fone, D., S. Hollinghurst, M. Temple, A. Round, N. Lester, A. Weightman, K. Roberts, E. Coyle, G. Bevan and S. Palmer. 2003. Systematic review of the use and value of computer simulation modelling in population health and health care delivery. Journal of Public Health Medicine 25:325-335.

Gaba, D. M. 2004. The future vision of simulation in health care. Quality and Safety in Health Care 13:i2-i10.

Graban, M. 2008. Lean Hospitals: Improving Quality, Patient Safety, and Employee Satisfaction. New York, Productivity Press.

Green, D. and T. Young. 2008. Value propositions for information systems in healthcare. In Proceedings of the 4lst Hawaii International Conference on System Sciences: 257-265. Hawaii, Institute of Electrical and Electronics Engineers, Inc.

Harper, P. R. and M. A. Pitt. 2004. On the challenges of healthcare modelling and a proposed project life cycle for successful implementation. The Journal of the Operational Research Society 55:657-661.

Hoot, N. R., L. J. LeBlanc, I. Jones, S. R. Levin, C. Zhou, C. S. Gadd and D. Aronsky. 2008. Forecasting emergency department crowding: a discrete event simulation. Annals of Emergency Medicine 52:116-125.

Jahangirian, M., S. C. Brailsford, T. Eldabi, P. Harper, A. Naseer, B. Patel, L. K. Stergioulas and T. Young. 2009. CrossSector Analysis of Simulation Methods: A Survey of Commerce, Defense and Healthcare. In Proceedings of the 21 st conference of Helenic Operational Research Society: Decision-Making In Healthcare Systems Athens, Greece.

Jahangirian, M., T. Eldabi, A. Naseer, L. K. Stergioulas and T. Young. Article in Press. Simulation in manufacturing and business: A review. European Journal of Operational Research doi:10.1016/j.ejor.2009.06.004.

Jun, J. B., S. H. Jacobson and J. R. Swisher. 1999. Application of discrete-event simulation in health care clinics: A survey. Journal of the Operational Research Society 50:109-123.

Kumar, A. and S. J. Shim. 2007. Eliminating emergency department wait by BPR implementation. In Proceedings of the 2007 IEEE International Conference on Industrial Engineering and Engineering Management 1679-1683. Singapore, Institute of Electrical and Electronics Engineers, Inc.

Locker, T. E. and S. M. Mason. 2005. Analysis of the distribution of time that patients spend in emergency departments. British Medical Journal 330:1188-1189. 
Mayhew, L. and D. Smith. 2008. Using queuing theory to analyse the Government's 4-h completion time target in Accident and Emergency departments. Health Care Management Science 11:11-21.

Morris, Z. S. and P. J. Clarkson. 2009. Does social marketing provide a framework for changing healthcare practice? Health Policy 91:135-141.

Naseer, A., T. Eldabi and M. Jahangirian. 2009. Cross-sector analysis of simulation methods: a survey of defense and healthcare. Transforming Government: People, Process and Policy 3:181-189.

Naseer, A., T. Eldabi and T. Young. Accepted. RIGHT: A toolkit for selecting healthcare modelling methods. Journal Of Simulation.

Pidd, M. and S. Robinson. 2007. Organising insights into simulation practice. In Proceedings of the 39th Conference on Winter Simulation: 40 Years! the Best Is Yet To Come 771-775. Washington D.C., Winter Simulation Conference, IEEE Press, Piscataway, NJ,.

Rosen, K. R. 2008. The history of medical simulation. Journal of Critical Care 23:157-166.

Rosen, S. P. 1991. Winning the next war. Ithaca, NY, Cornell University Publishing.

Royston, G. 2005. Modelling and simulation in health: potential achievement and challenge Available at http://www.pms.ac.uk/mashnet/pages/pdf/GeoffRoystonPresentation_MashnetLaunch.pdf [Accessed June 12th 2009]

Tunnicliffe Wilson, J. C. 1981. Implementation of computer simulation projects in health care. Journal of the Operational Research Society 32:825-832.

\section{AUTHOR BIOGRAPHIES}

TERRY YOUNG is Professor at the School of Information Systems, Computing and Mathematics (Brunel University). Terry first used numerical methods to model photonic devices and circuits in the 1980s and won the IEE Premium in 1989 for a paper on Finite Elements. In 2001 he joined Brunel as Chair of Healthcare Systems and his research interests centre on the organizational and technological aspects of the delivery of healthcare and the interplay between the two. His is the Principal investigator of MATCH (www.match.ac.uk). Email: <terry. young@brunel.ac.uk>

JULIE EATOCK is a Research Fellow on the MATCH project at Brunel University, UK. She holds a BSc in Mathematics from the University of Kent at Canterbury and an MSc(Eng) in Operational Research from the University of Birmingham. She worked as an Operational Research Analyst for Kent County Constabulary, before returning to academia to complete her $\mathrm{PhD}$ in simulation modeling. Her current research interests are business process modeling, in particular simulation modeling within the healthcare environment. Email: <Julie.Eatock@brunel.ac.uk>

MOHSEN JAHANGIRIAN is a Research Fellow working on the RIGHT project at the School of Information Systems, Computing and Mathematics in Brunel University, West London, UK. He received his PhD from University of Manchester on the field of Intelligent Simulation with an application in machine scheduling. Dr. Jahangirian has over 5 years of teaching experience in Management and Information Systems. His research interests include Modeling and Simulation, Information Systems, Artificial Intelligence, and OR. Email: <mohsen.jahangirian@brunel.ac.uk>

AISHA NASEER is a Research Fellow in the Department of Information Systems and Computing at Brunel University, West London, UK. She is working on the RIGHT project, funded by the EPSRC. She received her PhD in Semantic Integration of Heterogeneous Data Resources on HealthGrids from Brunel University. Her research interests include semantic interoperability, intelligent data management, health informatics, HealthGrid applications, Grid computing, Modeling and Simulation and artificial intelligence. Email: <aisha.naseer@brunel.ac.uk>

RICHARD LILFORD was Professor of obstetrics and gynecology in Leeds for 11 years before joining the Department of Health as a senior civil servant. He then joined the University of Birmingham as professor of clinical epidemiology and is now director of the Birmingham Clinical Research Academy and Head of the School of Health and Population Sciences. His interests are in research methodology, medical ethics and Bayesian statistics. He directs the NIHR Birmingham and Black Country CLAHRC, A $£ 20 \mathrm{M}$ evaluation of service delivery interventions, and he is a senior NIHR fellow. Email: $<r \cdot j \cdot l i l f o r d e b h a m \cdot a c \cdot u k>$ 